in the molecule, and it was suggested that this catalysis might occur in some enzyme-catalysed hydrolyses.

The next three papers dealt with the intimate details of the interactions between solvent and solute molecules. Dr. P. A. M. Wyatt of the University of Sheffield discussed the possibility of accounting for the properties of a solution in terms of a specific or 'chemical' solvation. $\mathrm{He}$ quoted as examples the thermal properties of solutions in sulphuric and nitric acids, and pointed out that, although solvation is important, other factors have to be considered, and that their nature is not well understood. Dr. J. W. Smith of Bedford College, London, showed how measurements of dielectric constant can give evidence for hydrogen-bonding in liquid mixtures, and how steric hindrance in aromatic amines could affect their interaction with solvent molecules. A semiempirical treatment for the dependence of the rates of nucleophilic substitutions upon solvent properties was outlined by Dr. R. F. Hudson of Queen Mary College, London, who applied this treatment to the kinetics of alcoholyses of acyl halides in solvents of low polarity.

Spectrophotometric methods for investigation of carbonium ions in non-aqueous solvents were described by Prof. A. G. Evans of University College, Cardiff. His group has studied the formation of these ions from triaryl compounds, and it seems that there are specific interactions between the phenol molecules of the solvent and the carbonium ions. Similar measurements have been made on the carbonium ions formed by protonation of an olefin, or by the reaction between an alkyl halide and a metal halide. During discussion of this work Dr. R. H. Plesch suggested that these spectrophotometric measurements could be used to calculate approximate values for the solvation energies of carbonium ions.

The chair was taken by Prof. M. S. Newman of the University of Ohio during the afternoon session. An electrostatic theory for the kinetic effects of solvents was presented by Prof. E. S. Amis of the University of Arkansas, who suggested that the rates of many reactions could be correlated simply with the bulk dielectric constant of the solvents. Subsequent speakers showed that this simple electrostatic theory could not account for many of the complex solvent effects on rates and equilibria.

Dr. H. Zollinger read a paper by himself and Dr. O. A. Stamm on the kinetics of diazo-coupling reactions studied in the University of Basle. They found that kinetic salt and solvent effects could only be considered in terms of specific interactions between ions or solvent molecules and the groups present in the reactants. The authors were able to interpret the effects of Brönsted bases on the rates and chemical course of these reactions by considering their mechan. istic details.

The first of two papers on solvent effects in nonpolar reactions was given by Dr. J. E. Leffler of Florida State University, who outlined the form of the interactions between ions or solvent molecules and the reactants and transition states, and described a very detailed kinetic study of the rates of racemizations of a number of optically active diphenyls. Dr. G. A. Russell of the General Electric Corporation gave results on the photochemical chlorination of aliphatic hydrocarbons in conditions such that the first step of the reaction is the abstraction of a hydro. gen atom from the hydrocarbon. It was possible to use the variation of product composition with changes in the solvent as evidence for the formation of a complex between an electrophilic chlorine atom and an aromatic hydrocarbon. In discussing this work Dr. W. A. Waters showed that while some free atoms and radicals had electrophilic properties others, such as sodium atoms, could act as nucleophilic reagents. The paper by Prof. L. F. Hatch and his co-workers described a re-investigation which they had made of the products of the addition of bromine to butadiene in various solvents.

The wide range of work discussed at this symposium shows the interest which chemists are taking in the effects of solvent composition upon rates and mechanisms. Understanding of the short-range forces which govern the interactions between solvent molecules and solutes during the collisions in which chemical reactions occur is now needed. It was therefore unfortunate that the recent work on nucleophilic substitutions in benzene, a solvent in which these interactions have been studied, was not one of the subjects under discussion.

C. A. Bunton

\section{BLOCK AND GRAFT COPOLYMERS}

CONTINUING earlier seminars on polymer (A science in the Chemistry Department of University College, London (cf. Nature, 178, 18 ; 1956), five lectures on block and graft copolymers were given on July 15.

Prof. M. Szware (Syracuse University, New York) described experiments which show that anionic polymerization of certain vinyl monomers, such as styrene, carried out under appropriate conditions, does not involve a termination step (cf. Szware and collaborators, J. Amer. Chem. Soc., 79, 2026 ; 1957 ; and references to earlier communications given there). Polymers, formed in this manner, are able to continue their growth, if a fresh portion of monomer is added, after the complete conversion of the original monomer. If a monomer, $B$, is added to a block of 'living' polymer $A \ldots A$ a block copoly. mer is formed. The original polymer $A \ldots A$ may possess one or two 'living' ends, and therefore a variety of block copolymers can be prepared by relatively simple experimental techniques. The interaction of 'living' polymers with proton donors, carbon dioxide and oxygen was shortly discussed.

Dr. C. H. Bamford (Courtaulds, Ltd.) described a method for the preparation of block copolymers. A monomer, $A$, carrying a teritiary base residue, is converted into a polymer, $A \ldots A-\underbrace{\mathrm{Me}}_{\mathrm{N}(\mathbf{E t})_{2}}-\mathrm{H}$, which is afterwards used as a macromolecular transfer agent in the polymerization of a second monomer, $B$. Chain transfer to the terminal group of the first polymer results in the formation of a block copolymer $A \ldots A-B . . B$. In a second method for preparing block copolymers, use is made of the following chain initiator : 
<smiles>CC(C#N)(CCC(=O)O)N=NC(C)(C#N)CCC(=O)O</smiles>

which leads to polymers containing terminal carboxyl groups. These latter species are coupled together by diols or other suitable bifunctional reagents. These two techniques were successfully applied for the preparation of block copolymers containing methylmethacrylate and either acrylonitrile or styrene. The composition and size distribution of some of these block copolymers could be calculated.

Drs. P. W. Allen, G. Ayrey and D. T. Turner (British Rubber Producers Research Association) gave lectures dealing with graft copolymers (I), made from natural rubber and polymethylmethacrylate. The copolymer (I) was prepared by the polymerization of methylmethacrylate, initiated by $\gamma$-radiation, this reaction being carried out in the presence of natural rubber. The influence of the purity of the natural rubber and of the methylmethacrylate, field intensity, concentration of methylmethacrylate and other variables on the rate of formation, yield and structure of the copolymer (I) were investigated. An alternative method of preparing the copolymer (I) consists in the benzoylperoxide initiated polymerization of methylmethacrylate, this process being again carried out in the presence of natural rubber. If azoisobutyronitrile is used as initiator, polymethylmethacrylate is formed, but no copolymer $(\mathbf{I})$. The cause of this specific initiator effect and some aspects of the mechanism have been investigated by using initiators labelled with carbon-14. In these investigations the separation of copolymer (I) from the natural rubber and methylmethacrylate was carried out by fractional precipitation, fractional extraction and turbidimetric titration methods. For purposes of characterization of (I) its molecular weight was determined, and after degradation of the rubber component with ozone, the molecular weight of the remaining polymethylmethacrylate component was measured.

\section{ELECTRONIC INSTRUMENTS EXHIBITION, MANCHESTER}

$\mathrm{T}$

HE Institution of Electronics is a nation-wide organization, but its headquarters are now in the north of England and its main centre of activity is Manchester, where the exhibition of electronic instruments organized by the Northern Division has, since the War, become established as an annual event. The twelfth annual Electronics Exhibition and Convention was held during July 11-17 at the Manchester College of Science and Technolog. About sixty manufacturers and other organizations exhibited their products, and the Exhibition was attended by about 14,000 visitors. Most of the exhibits consisted of commercially available equipment, which was in some cases shown in action, and there was also a special section for equipment still in the research stage. A programme of lectures and films ran concurrently with the Exhibition, and these were well attended.
Earlier Exhibitions have included a number of sections which would appeal to a wide public--for example, sound-reproducing equipment and electronic musical instruments. However, these were absent from this year's Exhibition, which was clearly aimed, even more than usual, at the professional user, and perhaps the manufacturer, of electronic instruments. For the latter, components and even such things as racks and chassis were shown by several firms. The Exhibition included an impressively wide range of instruments, of which a few only, a personal selection, can be mentioned. There was, in any event, little that was really new, although there were some items which had appeared since last year's Exhibition.

A flying-spot microscope which was shown by Cinema-Television, Ltd., is fitted with arrangements for counting the number of particles in its field, and their size distribution is obtained by successive counts with different adjustments of a size discriminator. A magnetic 'memory' in the counting unit ensures that a particle large enough to be scanned on several successive lines is only counted once: this is only defeated by particles the shape of which is re-entrant in the scanning direction, or perforated.

Another exhibit by Cinema-Television, Ltd., was a photographic contact printer designed to produce satisfactory contact prints from patchy negatives. A rather large spot of light from a cathode-ray tube scans, raster-wise, the negative with the printing paper in contact. The light passing through them both falls on a photocell the output of which is used to produce limited variations in the brightness of the spot. The broad scanning lines produced by the large spot overlap considerably, so that the definition is that of the negative and no scanning lines appear on the print. The instrument, in fact, carries out automatically the hand-shading usually employed in the printing of uneven negatives. Its cost, more than $£ 900$, will presumably limit it to large-scale users, and examples were given of its use in connexion with aerial photography and the commercial printing of amateurs' negatives. Another version which will make enlargements is to be produced.

An exhibit which was at least new to these Exhibitions was a version of the Land camera made by Langham Thompson, Ltd. In its present form it is intended for photographing cathode-ray tube displays and is fitted with an $f / 3.5$ lens and a singlespeed shutter. The novelty is that instead of film it uses special photographic paper with pods of developing and fixing chemicals attached to it at regular intervals. When the paper is wound on after exposure, rollers burst one of the pods and the chemicals are spread on the paper while it is still inside the camera. After waiting for one minute the paper is pulled out and emerges as a finished positive print. The material available is very fast ( $35^{\circ}$ Scheiner) and some twice as fast is promised soon. The operating cost is about $2 s .6 d$. a print (4 in. $\times 3$ in.).

Of the many cathode-ray oscilloscopes on show, the most novel were probably those by Cossor, Ltd., which are available only as kits of parts, but at little more than half the cost of comparable finished instruments. They use printed circuits throughout, and the made-up samples on show were clean-looking jobs-in fact, one suspects that the relative absence of wiring will greatly reduce the tangled web which sometimes results from amateur construction, as well as making the circuit characteristics more predictable. 